\title{
Competitive Online Scheduling with Level of Service
}

\author{
Ee-Chien Chang * \\ Department of Computational Science \\ National University of Singapore
}

\author{
Chee Yap * \\ Department of Computer Science \\ Courant Institute, New York University \\ July 7, 1998 (Revised: Oct 13, 2000)
}

\begin{abstract}
Motivated by an application in thinwire visualization, we study an abstract on-line scheduling problem where the size of each requested service can be scaled down by the scheduler. Thus our problem embodies a notion of "Level of Service" that is increasingly important in multimedia applications. We give two schedulers FirstFit and EndFit based on two simple heuristics, and generalize them into a class of greedy schedulers. We show that both FirstFit and EndFit are 2-competitive, and any greedy scheduler is 3-competitive. These bounds are shown to be tight.
\end{abstract}

\section{Introduction}

We study an abstract on-line scheduling problem motivated by visualization across a "thinwire" network $[4,3]$. An example of such a visualization problem is a server-client model where the server and client are connected by a thinwire (that is, a bandwidthlimited connection such as the Internet), with the the server holding a very large image that the client wishes to visualize. The viewer on the client side can control the transmission process by moving a mouse cursor over a low-resolution copy of the image to be visualized. This mouse motion generates, in real-time, a sequence of sampled positions along the mouse cursor trajectory. Each sampled position $(x, y)$ corresponds to a request for higher resolution data at the position. As the bandwidth is limited, we

*Partially funded by NSF grant CCR-9619846 
could only partially serve each request. This is where an on-line scheduler is needed to optimize the decisions. In most scheduling problems, a partially served request does not contribute to the performance of the scheduler. However, in this problem, a partially sent data can still provide useful information to the user. Thus, instead of sending all the requested data, the server has the option of lowering the "level" of the requested service in order to gain an overall better response time. This paper focuses on this level of service property. Note that there is considerable interest in similar Quality of Service (QoS) issues in multimedia research.

We use the standard notion of "competitiveness" in the sense of Sleator and Tarjan [9] to judge the quality of our online schedulers. A scheduler $S$ produces a feasible schedule $S(I)$ for each instance $I$ of our scheduling problem. Each $S(I)$ has an associated merit, where merit $(S(I)) \geq 0$. Let opt $(I)$ denote any feasible schedule for $I$ that maximizes the merit. We say $S$ is $c$-competitive $(c \geq 1)$ if for all $I$,

$$
\operatorname{merit}(\operatorname{opt}(I)) \leq c \cdot \operatorname{merit}(S(I))+b \text {, }
$$

where $b$ is a fixed constant. The competitive ratio of $S$ is defined by

$$
C(S):=\sup _{I} \frac{\operatorname{merit}(\operatorname{opt}(I))}{\operatorname{merit}(S(I))} .
$$

Thus, we want schedulers $S$ with $C(S) \geq 1$ as small as possible. The original paging problem studied by Sleator and Tarjan is a special case of the $k$-server problem [7]. There is a fairly large literature on competitive algorithms (e.g., [1, 2, 8]). The class of problems most closely related to ours is the online interval packing problem for a single server, where a schedule is a subset of non-overlapping intervals. Lipton and Tomkins [6] study a variant where the input intervals are sorted by their left endpoints. They give a randomized scheduler that is 2-competitive. As we will see, our problem is different from theirs in several ways. Woeginger [10] studied a problem that has several of the features of our problem. Other online interval packing problems can be found in $[11,5]$.

Outline. In the next section, we formulate our on-line scheduling problem and give two schedulers, FirstFit and EndFit. FirstFit is based on a heuristic which always serves the most important residual request, whereas EndFit is based on another heuristic which always serves according to the optimal schedule of the residual requests. The 2-competitiveness of FirstFit is shown in Section 3. A considerably harder result is the 2-competitiveness of EndFit, shown in Sections 4. We generalize both schedulers to a class of Greedy schedulers in Section 5. A lower bound of 1.17 on the competitive ratio of any deterministic scheduler is shown in Section 6. In section 7, we give a variant of the scheduling problem under a multi-tasking environment, and discuss its relationship with the original scheduling problem. 


\section{Problem Formulation}

We formalize our problem as an on-line scheduling problem. Each request $q$ has four parameters

$$
q=(s, t, v, w)
$$

where $s$ the start time, $t$ the termination time (or deadline), $v$ is the volume (or size), and $w$ is the weight. We require

$$
v \geq 0 \text { and } w \geq 0 \text {. }
$$

Write $\operatorname{st}(q), \operatorname{dl}(q), \operatorname{sz}(q), \operatorname{wt}(q)$ for the above parameters of $q$, respectively. Call the half-open interval $(s, t]$ the span of $q$, written $\operatorname{span}(q)$. A request $q$ can only be served within its $\operatorname{span}(s, t]$, and at any time moment $t_{0}$, at most one request can be served.

An instance $I$ is a sequence $\left(q_{1}, q_{2} \ldots q_{n}\right)$ of requests where the start times of the $q_{i}$ 's are in increasing order: note that we allow $\operatorname{st}\left(q_{i}\right)=\operatorname{st}\left(q_{i+1}\right)$ even though we nominally say $q_{i}$ starts before $q_{i+1}$. How requests are served is described by the schedule. Formally, A schedule for $I$ is a piece-wise constant function

$$
H: \mathbb{R} \rightarrow\left\{q_{1}, q_{2}, \ldots, q_{n}\right\} \cup\{\emptyset\}
$$

where $\left|H^{-1}\left(q_{k}\right)\right| \leq \mathbf{s z}\left(q_{k}\right)$ and $H^{-1}\left(q_{k}\right) \subseteq \operatorname{span}\left(q_{k}\right)$ for $k=1, \ldots, n$. Intuitively, $H\left(t_{0}\right)=q_{k}$ means the $k$ th request is served at time $t_{0}$ and $H\left(t_{0}\right)=\emptyset$ means no request is being served. A time moment $t_{0}$ is called a breakpoint if $H$ is discontinuous at $t_{0}$. (More precisely, for every $\varepsilon>0$, there exists $\delta_{i}\left(0<\delta_{i}<\varepsilon, i=1,2\right)$ such that $\left.H\left(t_{0}\right)=H\left(t_{0}-\delta_{1}\right) \neq H\left(t_{0}+\delta_{2}\right)\right)$. We further require a schedule $H$ to have finitely many breakpoints. A half-open interval of the form $\left(t_{0}, t_{1}\right]$ is called a timeslot. Without loss of generality, we may assume $H^{-1}(q)$ is a finite union of time slots. The merit merit $(H)$ of a schedule is

$$
\sum_{j=1}^{n} \operatorname{wt}\left(q_{j}\right)\left|H^{-1}\left(q_{j}\right)\right| .
$$

Relative to a schedule $H$ at any time $t_{0}$, we call

$$
v^{\prime}:=\operatorname{sz}(q)-\left|H^{-1}(q) \cap\left(-\infty, t_{0}\right]\right|
$$

the residual size of $q$. A request $q$ is completely served if $v^{\prime}=0$. If $v^{\prime}>0$ and $\operatorname{st}(q) \leq t_{0} \leq \mathrm{dl}(q)$, then we say $q$ is pending. The residue of a pending $q$ at time $t_{0}$ is the modified request $q^{\prime}=\left(t_{0}, \mathrm{dl}(q), v^{\prime}\right.$, wt $\left.(q)\right)$.

So for each completely served request, the scheduler gains sz $(q)$ wt $(q)$ merit points (so weights are multiplicative). Moreover, partially served requests gains a proportional fraction of this merit. This is unlike usual scheduling problems in which partially served requests receive no merit. 
Preemption. It is implicit in the above definitions that the servicing of any request can be preempted as often as we like with no penalty. Hence we may imagine the scheduler to "plan" a schedule based on all the currently residual requests. It services the requests according to this plan until the arrival of a new request. Then it suspends the current plan, recomputes a new plan based on the new set of residual requests, and repeats the process.

\section{$2.1 \quad$ Optimal Schedules}

We say $H$ is optimal for $I$ if merit $(H)$ is maximum among all schedules for $I$. The existence of optimal schedules is not immediate. In this section, we first establish the existence of optimal schedules (Theorem 2), and then give a canonical representation.

Let $I$ be a sequence of $n$ requests. The $2 n$ endpoints of intervals in $I$ are called natural breakpoints of $I$. The time-slot $(s, t]$ defined by two distinct consecutive natural breakpoints $s$ and $t$ is called a natural slot of $I$. There are at most $2 n-1$ natural slots.

Lemma 1 For every schedule $H$ for $I$, there is another schedule $H^{\prime}$ for $I$ with at most $2 n^{2}+n$ breakpoints such that:

- $\operatorname{merit}(H)=\operatorname{merit}\left(H^{\prime}\right)$;

- Within each natural slot, if a request is serviced, it is served in a single time interval.

Proof. We transform $H$ to $H^{\prime}$ by modifying its service within each natural slot of $I$. Within a natural slot, $H^{\prime}$ can always serve each request in a single (continuous) time interval. It follows that there are at most $n$ breakpoints within a natural slot. Some of the natural breakpoints may now become breakpoints of $H^{\prime}$. Since there are at most $2 n$ natural breakpoints and at most $2 n-1$ natural slots, $H^{\prime}$ has at most $2 n+(2 n-1) n=2 n^{2}+n$ breakpoints. Finally, note that the merit of $H$ is not changed by this transformation.

Q.E.D.

Theorem 2 For all sequences I of $n$ requests, there exists an optimal schedule $H^{*}$ with at most $2 n^{2}+n$ breakpoints.

Proof. In the following, let $\operatorname{slot}(i)$ denote the $i$-th natural slot where $\operatorname{slot}(1)$ is the earliest natural slot.

Let $\mu^{*}=\sup _{H}\{\operatorname{merit}(H)\}$ where $H$ range over all schedules for $H$. Then there is an infinite sequence $\left\{H_{k}: k \geq 0\right\}$ of schedules for $I$ such that merit $\left(H_{k}\right)$ is nondecreasing in $k$ and achieves $\mu^{*}$ in the limit. We now construct a schedule $H^{*}$ with $\operatorname{merit}\left(H^{*}\right)=\mu^{*}$. For any schedule $H$ for $I$, define the function

$$
\tau_{H}:\{1, \ldots, 2 n-1\} \times\{1, \ldots, n\} \rightarrow \mathbb{R}
$$


where $\tau_{H}(i, j)=\left|\operatorname{slot}(i) \cap H^{-1}\left(q_{j}\right)\right|$ where $q_{j}$ is the $j$-th request in $I$. Also, write $\tau_{k}$ instead of $\tau_{H_{k}}$. By going to a subsequence if necessary, we can assume that for all $i, j, \tau_{k}(i, j)$ is monotone (increasing or decreasing) as $k \rightarrow \infty$; we let $\tau^{*}(i, j)$ denote the limiting value. Choose $H_{k}^{\prime}$ to be the schedule obtained from $H_{k}$ using the transformation of the preceding lemma. Write $\tau_{k}^{\prime}$ instead of $\tau_{H_{k}^{\prime}}$. Finally, define $H^{*}$ so that $H^{*}$ has the form given by the previous lemma, and $\tau_{H^{*}}(i, j)=\tau^{*}(i, j)$ for all $i, j$. Clearly merit $\left(H^{*}\right)=\mu^{*}$. It remains to show that $H^{*}$ is a schedule for $I$. Fix an arbitrary $q_{j}$ in $I$. It suffices to check that $\left|\left(H^{*}\right)^{-1}\left(q_{j}\right)\right| \leq \mathbf{s z}\left(q_{j}\right)$. For all $k$, we have $\left|\left(H_{k}^{\prime}\right)^{-1}\left(q_{j}\right)\right|=\sum_{i=1}^{2 n-1} \tau_{k}^{\prime}(i, j) \leq \mathbf{s z}\left(q_{j}\right)$. Hence $\left|\left(H^{*}\right)^{-1}\left(q_{j}\right)\right|=\sum_{i=1}^{2 n-1} \tau^{*}(i, j) \leq \mathbf{s z}\left(q_{j}\right)$.

Q.E.D.

Ordering of Requests. The schedulers in this paper make decisions by giving priority to heavier weighted requests. In case $w t(p)=w t(q)$, we resolve the tie by treating $p$ as "heavier" than $q$ if and only if $p$ starts before $q$.

Canonical Schedule. A schedule $H$ is canonical if, the following hold:

P1. Serve lighter requests before heavier ones if possible. If $t_{0}<t_{1}$ and $H\left(t_{0}\right)$ is heavier than $H\left(t_{1}\right)$, then $d l\left(H\left(t_{0}\right)\right)<t_{1}$ or $\operatorname{st}\left(H\left(t_{1}\right)\right)>t_{0}$.

P2. Local Optimality. For all $t_{0}$ and $q_{1}$, if $q_{0}=H\left(t_{0}\right)$ is lighter than $q_{1}$, and $t_{0} \in$ $\operatorname{span}\left(q_{1}\right)$, then $\left|H^{-1}\left(q_{1}\right)\right|=s z(q)$.

In the above definition, if $H(t)=\emptyset$, we interpret $\emptyset$ to be the request $(-\infty,+\infty, \infty, 0)$. By definition, $\emptyset$ is lightest among all requests.

Lemma 3 Every schedule $H$ can be transformed into a canonical schedule $H^{\prime}$ where $\operatorname{merit}(H) \leq \operatorname{merit}\left(H^{\prime}\right)$.

Proof. First, we can modify $H$ so that it satisfies Lemma 1 and there are no violations of P2. After this, we keep performing "swaps" to remove violation of P1. Our swaps can be specified by two slots $T_{0}=\left(s_{0}, t_{0}\right]$ and slot $T_{1}=\left(s_{1}, t_{1}\right]$ where $s_{0}<t_{0} \leq s_{1}<t_{1}$. $H$ is constant each $T_{i}$, and they represent a violation of P2. We modify $H$ by serving $H\left(t_{1}\right)$ in $T_{0}$ and vice-versa. Such a swap does not create any new violations of Lemma 1 after suitable rearrangement within each natural slot. There is also no new violation of $\mathrm{P} 2$, and the merit does not change. We choose each swap so that $t_{1}$ is maximized, and subject to this, we also maximize $t_{1}-s_{1}$. So in successive swaps, $t_{1}$ will strictly decrease. If this process stops after finitely many steps, then $H^{\prime}$ can be chosen to be the final schedule. Otherwise, by a limiting type argument similar to the proof of Lemma 1, we can construct a canonical schedule $H^{\prime}$ from this infinite sequence of schedules.

Q.E.D.

In particular, if $H$ is optimal, $H^{\prime}$ will be a canonical optimal schedule. Let opt $(I)$ denote any canonical optimal schedule for $I$. 


\section{$2.2 \quad$ FirstFit}

The online scheduler that always serve the heaviest residual request at each moment is called the FirstFit scheduler. Figure 1 shows the schedule produced by FirstFit on an instance of two requests $q_{1}$ and $q_{2}$. Although this example may appear contrived, we can modify $q_{2}$ to $\widetilde{q}_{2}$ where $\widetilde{q}_{2}=(0,2,1,1+\epsilon)$. As $\epsilon$ tends to zero, the FirstFit schedule will be the one shown in Figure 1.

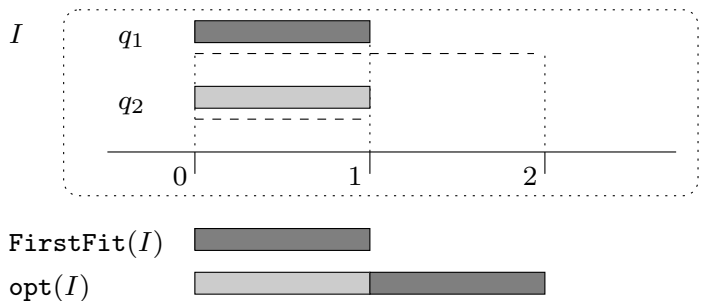

Figure 1: The top figure illustrates the instance $I$ of two requests: $q_{1}=(0,2,1,1)$ and $q_{2}=(0,1,1,1)$. Each horizontal "dashed" line represents the span of the request. Although wt $\left(q_{1}\right)=\operatorname{wt}\left(q_{2}\right), q_{1}$ is "heavier" than $q_{2}$ by our tie-breaking rule. In opt $(I)$, $q_{1}$ and $q_{2}$ are served in the time-slots $(1,2]$ and $(0,1]$, respectively. However, in FirstFit $(I)$, only $q_{1}$ is served.

\subsection{EndFit}

The Off-line EndFit Scheduler. Consider an online scheduler which always serves according to the optimal schedule for the current set of residual requests. This was first suggested by Estie Arkin. ${ }^{1}$ To implement such a scheduler, we can invoke a general off-line algorithm for computing optimal schedules upon each new arrival of a request. But it turns out that a very simple scheduler can be used. This scheduler, on an arbitrary instance $I$, operates as follows:

Starting from the heaviest request down to the lightest, allocates each request $q \in I$ to the latest available time-slot(s) within $\operatorname{span}(q)$.

Call this the OffEndFit scheduler. It is an off-line algorithm because it must see the entire set of requests to make its decisions. The next lemma shows that this scheduler is optimal for a special class of inputs.

Lemma 4 If $I$ is an instance in which all requests have a common starting time, then OffEndFit $(I)$ is an optimal schedule for I that is both canonical and unique.

\footnotetext{
${ }^{1}$ Private communication (1997).
} 
Proof. Let $H:=\operatorname{OffEndFit}(I)$ and $H^{*}:=\operatorname{opt}(I)$ be any canonical optimal schedule. First, observe that $H$ has canonical form and, of course, $H$ is uniquely determined by $I$. Hence it remains to show that $H^{*}$ is equal to $H$. By way of contradiction, let $\left(s_{1}, t_{1}\right]$ be a time slot such that $H$ and $H^{*}$ are constant on this slot but $q=H\left(t_{1}\right) \neq H^{*}\left(t_{1}\right)=$ $q^{*}$. We may assume that $t_{1}$ is maximized, so that for all $t>t_{1}, H(t)=H^{*}(t)$. By symmetry, we may assume that $q$ is heavier than $q^{*}$. By property $\mathrm{P} 2$ for $H^{*}$, we infer that that $q$ was served at some time before $t_{1}$. But this contradicts property P1. Hence $H=H^{*}$.

Q.E.D.

The online EndFit scheduler. Let EndFit be the online scheduler which always serves according to the OffEndFit schedule for the residual requests. More precisely, on arrival of a new request $q$, EndFit preempts the current service. It computes a new schedule $P$ for the current residual requests using OffEndFit, and continues by servicing $P$. Since all residual requests have a common starting time, $P$ is the canonical optimal schedule. We call $P$ the plan of EndFit upon the arrival of request $q$, and let $\operatorname{Plan}^{+}$(EndFit, $I, q$ ) denote the plan upon the arrival of $q$. In addition, let $\operatorname{Plan}^{-}$(EndFit, $\left.I, q\right)$ denote the plan just before the arrival of $q$. It is helpful to visualize the relationship between $\operatorname{Plan}^{-}$(EndFit, $\left.I, q\right)$ and $\operatorname{Plan}^{+}$(EndFit, $I, q$ ). To obtain $\operatorname{Plan}^{+}$(EndFit, $I, q$ ) from Plan $^{-}$(EndFit, $I, q$ ), the scheduler first finds the location to "insert" the newly started request $q$; it then "squeezes" $q$ in by "pushing" the original allocated requests leftward (to earlier time-slots). In so doing, some requests may be "pushed out" from the plan.

Figure 2 shows the EndFit schedule for an instance $I=\left(q_{1}, q_{2}\right)$. The EndFit schedule for this example may appear contrived. To see that it is "correct in the limit", let $I_{\epsilon}=\left(q_{0}, q_{1}, q_{2}\right)$ where $q_{0}=(0,1,1, \epsilon)$. For any $0<\epsilon<1$, the off-line optimal schedule for the current residual requests is unique. As $\epsilon \rightarrow 0$, EndFit $\left(I_{\epsilon}\right)$ approaches the one schedule shown in Figure 2.

In the special cases where each request $q$ in $I$ satisfies $\operatorname{span}(q)=\mathrm{dl}(q)$, we have $\operatorname{EndFit}(I)=\operatorname{FirstFit}(I)=\operatorname{opt}(I)$.

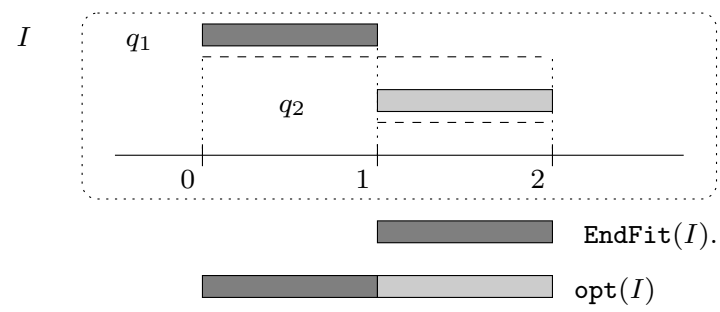

Figure 2: The top figure illustrates the instance $I$ of two requests: $q_{1}=(0,2,1,1)$ and $q_{2}=(1,2,1,1)$. In the opt $(I), q_{1}$ and $q_{2}$ are served. However, in EndFit $(I)$, only $q_{1}$ is served. 


\section{Competitive Ratio of FirstFit}

Example 1 (Figure 1) shows that the competitive ratio of FirstFit is at least 2. We will show that FirstFit is 2-competitive. Before presenting the proof, let us give two definitions.

Charging Scheme. Let $H, H_{1}$ and $H_{2}$ be schedules for an instance $I$. We often need to argue that the merit of $H$ is no larger than the sum of the merits of $H_{1}$ and $H_{2}$. Our approach is to charge a portion of $H$ to $H_{1}$ and the remaining to $H_{2}$. Intuitively, the charging process can be viewed as first cutting $H_{1}$ and $H_{2}$ into pieces and then piecing them together again to form another piecewise-constant function $H_{\text {chg }}$. Each piece, after cutting, may be translated before being placed in their slot in $H_{\text {chg }}$. As it may turn out that $\left|H_{\text {chg }}^{-1}(q)\right|>\mathbf{s z}(q)$ for some request $q, H_{\text {chg }}$ is not necessarily a schedule. The cut-and-paste is done in a way that for all $t, \operatorname{wt}\left(H_{\operatorname{chg}}(t)\right) \geq \operatorname{wt}(H(t))$. Therefore,

$$
\operatorname{merit}(H) \leq \operatorname{merit}\left(H_{\mathrm{chg}}\right) \leq \operatorname{merit}\left(H_{1}\right)+\operatorname{merit}\left(H_{2}\right) \text {. }
$$

In particular, if $H_{1}=H_{2}$, then we have

$$
\operatorname{merit}(H) \leq 2 \cdot \operatorname{merit}\left(H_{1}\right)
$$

When we use the phrase: "charge $\left(s^{\prime}, e^{\prime}\right]$ from $H$ to $H_{1}$ at $(s, e]$ ", we mean that a piece $(s, e]$ is cut from $H_{1}$ and placed into the slot $\left(s^{\prime}, e^{\prime}\right]$ in $H_{\text {chg }}$. Equivalently, if $H^{-1}(q)$ is the interval $\left(s^{\prime}, e^{\prime}\right]$, we may say that the request $q$ is charged from $H$ to $H_{1}$ at $(s, e]$ (see Figure 3). Thus, to show (1), we need to charge each time slot of $H$ to either $H_{1}$ or $H_{2}$, and ensure that no part of $H_{1}$ or $H_{2}$ is charged more than one.

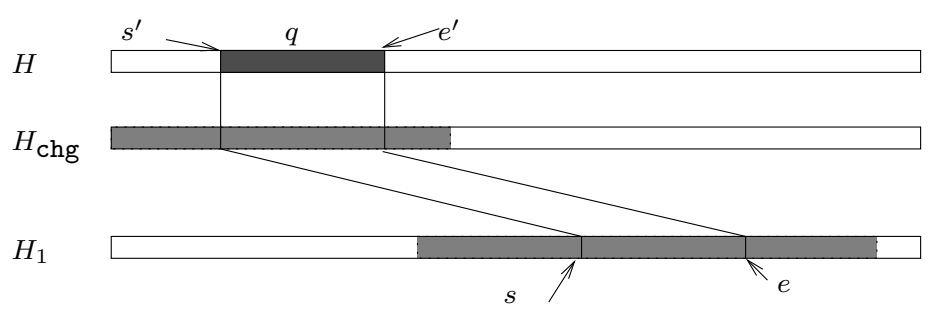

Figure 3: Charging the request $q$ in $H$ to $H_{1}$ at $(s, e]$.

Intactness. A request $q$ is intact in a schedule $H$ if $H^{-1}(q)$ is connected, and either $\left|H^{-1}(q)\right|=0$ or $\operatorname{sz}(q)$. An instance $I$ is intact in a schedule $H$ if each request $q$ in $I$ is intact. Most of our proofs will be simplified if we assume intactness. Fortunately, since our scheduling problem is preemptive, we could break a request into pieces so as 
to achieve intactness. For example, consider an instance $I$ consisting of one request $q=[0,5,4,1]$, and a schedule $H$ whereby $q$ is served in time slots $(0,2]$ and $(3,4]$. Thus, $q$ is not intact in $H$. Now, construct a new instance $I^{\prime}=\left\langle q_{1}, q_{2}, q_{3}\right\rangle$ by breaking $q$ into the following pieces:

$$
\begin{aligned}
& q_{1}=[0,5,2,1], \\
& q_{2}=[0,5,1,1], \text { and } \\
& q_{3}=[0,5,1,1] .
\end{aligned}
$$

Consider a scheduler $H^{\prime}$ in which $q_{1}$ and $q_{2}$ are served in time slot $(0,2]$ and $(3,4]$ respectively. The new schedule $H^{\prime}$ is essentially $H$, and the instance $I^{\prime}$ is intact in $H^{\prime}$. Note that a scheduler $S$ might behaves differently on $I$ and $I^{\prime}$. However, FirstFitand EndFitare well-behaved in the sense that FirstFit $(I)$ and EndFit $(I)$ are essentially the same as FirstFit $\left(I^{\prime}\right)$ and $\operatorname{EndFit}\left(I^{\prime}\right)$.

Theorem 5 For any instance I,

$$
\operatorname{merit}(\operatorname{opt}(I)) \leq 2 \cdot \operatorname{merit}(\text { FirstFit }(I)) \text {. }
$$

Proof.

Given an instance $I$, let $H_{\mathrm{ff}}:=\operatorname{FirstFit}(I)$ and $H_{\mathrm{opt}}:=\operatorname{opt}(I)$. We can assume that $I$ is intact in both $H_{\mathrm{ff}}$ and $H_{\mathrm{opt}}$.

Let $H_{0}$ be an identical copy of $H_{\text {ff }}$. We want to charge requests served in $H_{\text {opt }}$ to $H_{0}$ and $H_{\mathrm{ff}}$. Let $\left\{t_{1}, t_{2}, \ldots, t_{m}\right\}$ be the distinct breakpoints in $H_{\mathrm{opt}}$, where $t_{i}<t_{j}$ if and only if $i<j$.

For each $t_{i}$, starting from $i:=1$ to $m-1$, consider the time-slot $\left(t_{i}, t_{i+1}\right]$. Let $q_{\mathrm{opt}}:=H_{\mathrm{opt}}\left(t_{i+1}\right)$. If $q_{\mathrm{opt}}=\emptyset$, then let $q_{\mathrm{opt}}:=\left[t_{i}, t_{i+1},\left(t_{i+1}-t_{i}\right), 0\right]$. Let $q_{\mathrm{ff}}$ be the lightest request served during $\left(t_{i}, t_{t+1}\right]$ by FirstFit. If the lightest request does not exist, let $q_{\mathrm{ff}}:=\left[t_{i}, t_{i+1},\left(t_{i+1}-t_{i}\right), 0\right]$.

There are two cases:

1. If the request $q_{\mathrm{ff}}$ is not lighter than $q_{\mathrm{opt}}$, charge $q_{\mathrm{opt}}$ from $H_{\mathrm{opt}}$ to $H_{\mathrm{ff}}$ at $\left(t_{i}, t_{i+1}\right]$.

2. Otherwise, charge $q_{\text {opt }}$ from $H_{\text {opt }}$ to $H_{0}$ at $H_{0}^{-1}\left(q_{\text {opt }}\right)$.

We have to show that in the second case, $\left|H_{0}^{-1}\left(q_{\text {opt }}\right)\right| \geq\left|\left(t_{i}, t_{i+1}\right]\right|$. In the first place, why is the weight of $q_{\mathrm{ff}}$ lighter? The request $q_{\mathrm{ff}}$ is chosen by FirstFit because it is the heaviest request among the pending requests. This implies that $q_{\text {opt }}$ is not a pending request, even though $t_{i}$ is in the span of $q_{\text {opt }}$. So $q_{\text {opt }}$ must have been completely served by FirstFit. This implies that $\left|H_{0}^{-1}\left(q_{\text {opt }}\right)\right| \geq\left|\left(t_{i}, t_{i+1}\right]\right|$.

Q.E.D. 


\section{Competitive Ratio of EndFit}

Example 2 (Figure 2) shows that the competitive ratio of EndFit is at least 2. We now show that this constant is the best possible.

The upper bound proof is considerably more subtle than the proof for FirstFit in the last section. The key result is Theorem 11 below which formalizes this observation about EndFit: it never hurts the performance of EndFit to have a request started at an earlier time. For example, in Figure 2, the performance of EndFit will improve if the request $q_{2}$ starts at an earlier time. The analogous lemma fails for FirstFit. For example, in Figure 1, the performance of FirstFit would improve if $q_{1}$ starts at a time later than 0 .

\subsection{Trimmed instances and level sets}

A request $\widetilde{q}$ is a trimmed version of $q$ if $\operatorname{st}(\widetilde{q}) \geq \operatorname{st}(q), \operatorname{dl}(\widetilde{q})=\operatorname{dl}(q)$, $\operatorname{wt}(\widetilde{q})=\operatorname{wt}(\widetilde{q})$ and $\mathbf{s z}(\widetilde{q}) \leq \mathbf{s z}(q)$. Thus, a trimmed version of $q$ may start later than the original $q$. The main motivation for this definition comes from the fact that the residual request of any request $q$ is a trimmed version of $q$.

An instance $\widetilde{I}$ is a trimmed instance of $I$ if there is a one-one (not necessarily onto) mapping from $\widetilde{I}$ to $I$ such that any $\widetilde{q}$ in $\widetilde{I}$ is a trimmed version of its corresponding request in $I$. Clearly, merit $($ opt $(\widetilde{I})) \leq$ merit $($ opt $(I))$. We want to show that a similar relationship also holds for $\operatorname{EndFit}(\widetilde{I})$ and $\operatorname{EndFit}(I)$.

To analyze EndFit, we introduce the notion of "level sets". Let $H$ be a schedule for $I$ and $q$ be any request. We do not require $q$ to belong to $I$ but it must be totally ordered with respect to the requests in $I$. Define the $q$-level set of $H$ to be

$$
\operatorname{Level}_{H}(q):=\{t \in \mathbb{R}: \operatorname{wt}(H(t)) \geq \operatorname{wt}(q)\} .
$$

Also define the related sets,

$$
\operatorname{Level}_{H}^{+}(q):=\{t \in \mathbb{R}: \operatorname{wt}(H(t))>\operatorname{wt}(q)\}
$$

and

$$
\operatorname{Level}_{H}^{0}(q):=\{t \in \mathbb{R}: \operatorname{wt}(H(t))=\operatorname{wt}(q)\} .
$$

In these three notions of level sets, the comparison of weights are not really comparison of real numbers, but uses our tie-breaking conventions for weights of requests. E.g. "wt $(H(t))>\operatorname{wt}(q)$ " should really read "request $H(t)$ is heavier than request $q$ ". For this reason, we do not define "Level ${ }_{H}(w)$ " where $w$ is a real number because the real number $w$ does not carry any tie-breaking information.

Clearly, $\operatorname{Level}_{H}(q)=\operatorname{Level}_{F}^{+}(q) \cup \operatorname{Level}_{F}^{0}(q)$. In case $H$ is equal to $\operatorname{OffEndFit}(I)$, we will write $\operatorname{Level}_{I}(q), \operatorname{Level}_{I}^{+}(q), \operatorname{Level}_{I}^{0}(q)$ instead of Level ${ }_{H}(q), \operatorname{Level}_{H}^{+}(q), \operatorname{Level}_{H}^{0}(q)$, respectively. It is important that note that we use OffEndFit, not EndFit in this definition. 
We break up the operations of OffEndFit using the following device: by a level set $L$ we mean a finite union of time slots. If $q$ is a request, define $\operatorname{EF}(q, L) \subseteq \mathbb{R}$ to be the time slots that are scheduled for $q$ by the OffEndFit scheduler, assuming that $L$ have already been scheduled. Thus $\operatorname{EF}(q, L) \cap L$ is empty.

If $q \in I$, then

$$
\operatorname{Level}_{I}^{0}(q)=\operatorname{EF}\left(q, \operatorname{Level}_{I}^{+}(q)\right)
$$

Hence,

$$
\operatorname{Level}_{I}(q)=\operatorname{EF}\left(q, \operatorname{Level}_{I}^{+}(q)\right) \cup \operatorname{Level}_{I}^{+}(q) .
$$

Writing $s=\inf \operatorname{Level}_{I}^{0}(q)$, we can further express this as

$$
\operatorname{Level}_{I}(q)=(s, \operatorname{dl}(q)] \cup \operatorname{Level}_{I}^{+}(q) .
$$

In general, for any level set $L$ and any $q$, if $s=\inf \operatorname{EF}(q, L)$ then

$$
\mathrm{EF}(q, L) \cup L=(s, \mathrm{dl}(q)] \cup L
$$

Lemma 6 Let $L^{\prime} \subseteq L$ be level sets. If $\widetilde{q}$ is a trimmed version of $q$ then

$$
\mathrm{EF}\left(\widetilde{q}, L^{\prime}\right) \cup L^{\prime} \subseteq \mathrm{EF}(q, L) \cup L
$$

Proof. Let $C=\operatorname{span}(q) \backslash L$ and $C^{\prime}=\operatorname{span}(\widetilde{q}) \backslash L^{\prime}$. For any $s \in \mathbb{R}$, let $C_{s}=\{t \in$ $C: t>s\}$. Similarly define $C_{s}^{\prime} \subseteq C^{\prime}$. Because $\widetilde{q}$ is a trimmed version of $q$, for all $s \geq \operatorname{st}(\widetilde{q})$ (which is $\geq \operatorname{st}(q)$ ), we have

$$
C_{s} \subseteq C_{s}^{\prime}
$$

But $\operatorname{EF}\left(\widetilde{q}, L^{\prime}\right)=C_{s_{0}}^{\prime}$ for some $s_{0} \geq \operatorname{st}(\widetilde{q})$, and similarly $\operatorname{EF}(q, L)=C_{s_{1}}$ for some $s_{1} \geq \operatorname{st}(q)$. Here, we pick the largest possible value for $s_{0}$ and $s_{1}$. According to (2)

$$
\operatorname{EF}\left(\widetilde{q}, L^{\prime}\right) \cup L^{\prime}=\left(s_{0}, \operatorname{dl}(\widetilde{q})\right] \cup L^{\prime}, \quad \operatorname{EF}(q, L) \cup L=\left(s_{1}, \operatorname{dl}(q)\right] \cup L .
$$

Hence the lemma would follow if $\left(s_{0}, \mathrm{dl}(\widetilde{q})\right] \subseteq\left(s_{1}, \mathrm{dl}(q)\right]$. Since $\mathrm{dl}(q)=\mathrm{dl}(\widetilde{q})$, it is enough to show

$$
s_{1} \leq s_{0} .
$$

To see this, note that

$$
\left|C_{s_{0}}\right| \leq\left|C_{s_{0}}^{\prime}\right| \leq \mathrm{sz}(\widetilde{q}) \leq \mathrm{sz}(q)
$$

and

$$
s_{0} \geq \operatorname{st}(\widetilde{q}) \geq \operatorname{st}(q)
$$

using the fact that $\widetilde{q}$ is a trimmed version of $q$. If $\left|C_{s_{0}}\right|=\operatorname{sz}(q)$ or $s_{0}=\operatorname{st}(q)$ then we must have $s_{1}=s_{0}$ Otherwise, suppose $\left|C_{s_{0}}\right|<\mathbf{s z}(q)$ and $s_{0}>\operatorname{st}(q)$. Then it is immediate that $s_{1}<s_{0}$.

Q.E.D. 


\subsection{Domination}

In the following, let $\widetilde{I}$ be a trimmed version of $I$. Our goal is to prove that

$$
\operatorname{merit}(\operatorname{EndFit}(\widetilde{I})) \leq \operatorname{merit}(\operatorname{EndFit}(I))
$$

where $\widetilde{I}$ is a trimmed version of $I$. The key idea is to study the domination relation: for two schedules, we say that $H$ dominates $H^{\prime}$ if for all $t$, wt $(H(t)) \geq \operatorname{wt}\left(H^{\prime}(t)\right)$. For two input instances, we say $I$ dominates $I^{\prime}$ if OffEndFit $(I)$ dominates OffEndFit $\left(I^{\prime}\right)$. Again, note that domination between instances is defined in terms of OffEndFit, not EndFit. Domination can be reduced to inclusion relationship between level sets: thus, $I$ dominates $I^{\prime}$ if and only if for all $q^{\prime} \in I^{\prime}$,

$$
\operatorname{Level}_{I^{\prime}}\left(q^{\prime}\right) \subseteq \operatorname{Level}_{I}\left(q^{\prime}\right)
$$

Lemma 7 If $\widetilde{I}$ is a trimmed version of $I$ then $I$ dominates $\widetilde{I}$.

Proof. We use induction on the number of requests in $I$. Suppose $q$ is the lightest request in $I$ and $\widetilde{q} \in \widetilde{I}$ is the corresponding trimmed version of $q$. Just before OffEndFit schedules $q$, we have a partial schedule $H$ for all the requests in $I \backslash\{q\}$. Let $H^{\prime}$ be similarly defined with respect to $\widetilde{I}$ and $\widetilde{q}$. Let $L:=\operatorname{Level}_{I}^{+}(q)=\operatorname{Level}_{H}^{+}(q)$ and $L^{\prime}:=\operatorname{Level}_{\widetilde{I}}^{+}(\widetilde{q})=\operatorname{Level}_{H^{\prime}}^{+}(\widetilde{q})$. By induction, $H$ dominates $H^{\prime}$. To show that $I$ dominates $\widetilde{I}$, it remains to show

$$
\operatorname{Level}_{\widetilde{I}}(\widetilde{q}) \subseteq \operatorname{Level}_{I}(q) .
$$

$\operatorname{But~Level}_{\widetilde{I}}(\widetilde{q})=\operatorname{EF}\left(\widetilde{q}, L^{\prime}\right) \cup L^{\prime}$ and $\operatorname{Level}_{I}(w)=\operatorname{EF}(q, L) \cup L$. Since, $L^{\prime} \subseteq L$ and $\widetilde{q}$ is a trimmed version of $q$, Lemma 6 implies (4).

Q.E.D.

As corollary, if $\widetilde{I}$ is obtained by deleting a request from $I$, then $I$ dominates $\widetilde{I}$. Note that the preceding lemma does not depend on the requests in $I$ or $\widetilde{I}$ having a common start time.

Next, for any time $t \in \mathbb{R}$, let $\left.I\right|_{t}$ denote the residual instance of $I$ when the schedule EndFit $(I)$ has been serviced up to time $t$. More precisely, each $q \in I$ with $\operatorname{dl}(q) \leq t$ is deleted. Otherwise, $q$ is replaced by $q_{t}$ which is the residue of $q$ at time $t$ in EndFit $(I)$. If $q$ has been completely served by time $t$, then its residue has zero size, $\mathbf{s z}\left(q_{t}\right)=0$. Such requests are called null requests. For technical reasons, we do not discard null requests from $\left.I\right|_{t}$. Clearly all requests in $\left.I\right|_{t}$ starts at or after time $t$.

To motivate the next definition, observe that if $I$ dominates $\widetilde{I}$, then for all $t,\left.I\right|_{t}$ dominates $\left.\widetilde{I}\right|_{t}$. Unfortunately, $\left.\widetilde{I}\right|_{t}$ may no longer be a trimmed version of $\left.I\right|_{t}$, even if $\widetilde{I}$ is a trimmed version of $I$. For example, let $I=\left(q_{0}, q_{1}\right)=((0,3,2,1),(1,2,1,2))$ and $\widetilde{I}=\left(q_{0}^{\prime}, q_{1}\right)=((1,3,2,1),(1,2,1,2))$. If $t=1,\left.I\right|_{t}=\left(q_{2}, q_{1}\right)=((1,3,1,1),(1,2,1,2))$ while $\left.\widetilde{I}\right|_{t}=\left(q_{0}^{\prime}, q_{1}\right)=((1,3,2,1),(1,2,1,2))$. Then $\left.\widetilde{I}\right|_{t}$ is not a trimmed version of $\left.I\right|_{t}$ because $q_{0}^{\prime}$ is not a trimmed version of $q_{2}$. According to the definitions below, it turns out that $q_{2}$ "spans" $q_{0}^{\prime}$, and $q_{2}$ is "saturated" in $\left.I\right|_{t}$. 
A request $q \in I$ is saturated in $I$ if

$$
\left|\operatorname{span}(q) \backslash \operatorname{Level}_{I}^{+}(q)\right| \leq \mathbf{s z}(q)
$$

Note that $\operatorname{span}(q) \backslash \operatorname{Level}_{I}^{+}(q)$ comprise all the times available for scheduling $q$ relative to $I$. A request $q$ spans another request $q^{\prime}$ if $\operatorname{span}\left(q^{\prime}\right) \subseteq \operatorname{span}(q)$. For example, if $q^{\prime}$ is a trimmed version of $q$ then $q$ spans $q$. We define a binary relation " $\succeq$ " on instances as follows: if $I$ and $J$ are instances, we write

$$
I \succeq J
$$

if $I=\left(q_{1}, \ldots, q_{n}\right)$ and $J=\left(q_{1}^{\prime}, \ldots, q_{n}^{\prime}\right)$ and for all $i=1, \ldots, n, \operatorname{wt}\left(q_{i}\right)=\operatorname{wt}\left(q_{i}^{\prime}\right)$ and

(a) either $q_{i}^{\prime}$ is a trimmed version of $q_{i}$, or

(b) $q_{i}$ spans $q_{i}^{\prime}$ and $q_{i}$ is saturated in $I$.

If $f: I \rightarrow J$ such that $f\left(q_{i}\right)=q_{i}^{\prime}$ for all $i$, then we say " $I \succeq J$ via $f$ ". Note that if $J$ is a trimmed version of $I$ and $|I|=|J|$, then $I \succeq J$. The following is immediate from the definition of $\succeq$ :

Lemma 8 Let $f: I \rightarrow J$ be a bijection and $f(q)=\widetilde{q}$ for some $q \in I$. Let $I^{\prime}=I \backslash\{q\}$ and $J^{\prime}=J \backslash\{\widetilde{q}\}$ and $f^{\prime}: I^{\prime} \rightarrow J^{\prime}$ be the restriction of $f$ to $I^{\prime}$. If $I^{\prime} \succeq J^{\prime}$ via $f^{\prime}$ then $I \succeq J$ via $f$.

The next lemma shows that the relation $\succeq$ is preserved by residual instances:

Lemma 9 If $I \succeq J$ then for all $t,\left.\left.I\right|_{t} \succeq J\right|_{t}$.

Proof. Suppose $I \succeq J$ via $f$. Let $q \in I$ and $\widetilde{q}=f(q)$. There are two cases: (a) Suppose $\widetilde{q}$ is a trimmed version of $q$. Let $\widetilde{q}_{t}$ and $q_{t}$ be the corresponding requests in $\left.J\right|_{t}$ and in $\left.I\right|_{t}$. Clearly, $q_{t}$ spans $\widetilde{q}_{t}$. If $\mathbf{s z}\left(q_{t}\right) \geq \mathbf{s z}\left(\widetilde{q}_{t}\right)$ then $\widetilde{q}_{t}$ is a trimmed version of $q_{t}$. Otherwise, it must be the case that $q_{t}$ became saturated in $\left.I\right|_{t}$. (b) Suppose $q$ spans $\widetilde{q}$ and $q$ is saturated in $I$. Then it is easy to see that $q_{t}$ spans $\widetilde{q}_{t}$ and $q_{t}$ is saturated in $\left.I\right|_{t}$.

Q.E.D.

The next result connects the relation $\succeq$ to domination:

Lemma 10 If $I \succeq J$ then $I$ dominates $J$.

Proof. Let $I \succeq J$ via $f: I \rightarrow J$. We use induction on the number $|I|=|J|$ of requests in $I$ or $J$. Suppose $|I|=1$. In case condition (a) holds in the definition of $I \succeq J$, then clearly $I$ dominates $J$. In case condition (b) holds, then $q$ is saturated in $I$ implies $\mathrm{dl}(q)-\mathrm{st}(q)=\mathbf{s z}(q)$. Combined with the fact that $q$ spans $f(q)$, we conclude that $\mathbf{s z}(f(q)) \leq \mathbf{s z}(q)$. So again, $I$ dominates $J$. Next assume $|I|>1$ and let $q$ be the lightest request in $I$. So $f(q)$ is the lightest in $J$. Let $J^{\prime}=J \backslash\{f(q)\}$ 
and $I^{\prime}=I \backslash\{q\}$. It remains to prove that $\operatorname{Level}_{J}(f(q)) \subseteq \operatorname{Level}_{I}(q)$. By induction, $I^{\prime}$ dominates $J^{\prime}$. We have

$$
\operatorname{Level}_{J}^{+}(f(q))=\operatorname{Level}_{J^{\prime}}^{+}(f(q)) \subseteq \operatorname{Level}_{I^{\prime}}^{+}(q)=\operatorname{Level}_{I}^{+}(q)
$$

If $f(q)$ is a trimmed version of $q$, then it follows from (5) and Lemma 6 that Level $J_{J}(f(q)) \subseteq$ Level $_{I}(q)$. Otherwise, $q$ spans $f(q)$ and $q$ is saturated in $I$. This means

$$
\begin{aligned}
\operatorname{Level}_{I}(q) & \left.=\operatorname{Level}_{I}^{+}(q) \cup \operatorname{span}(q) \quad \text { (since } q \text { is saturated in } I\right) \\
& \left.\supseteq \operatorname{Level}_{J}^{+}(f(q)) \cup \operatorname{span}(f(q)) \quad \text { (since } q \text { spans } f(q)\right) \text { and by }(5) \\
& \supseteq \operatorname{Level}_{J}(f(q)) .
\end{aligned}
$$

Q.E.D.

We come to the main result.

Theorem 11 If $\widetilde{I}$ is a trimmed version of I then

$$
\operatorname{merit}(\operatorname{EndFit}(\widetilde{I})) \leq \operatorname{merit}(\operatorname{EndFit}(I)) \text {. }
$$

Proof. It sufficient to prove this result in the case where $I$ and $\widetilde{I}$ are identical except that some $q \in I$ is replaced by a trimmed version $\widetilde{q}$ in $\widetilde{I}$. Let

$$
s_{0} \leq s_{1} \leq \cdots \leq s_{m-1} \leq s_{m}, \quad(m \geq 0)
$$

be the sequence of start times of all $m+1$ requests in $I$. There are schedules $H_{0}, H_{1} \ldots H_{m}$ such that in the time slot $\left(s_{i}, s_{i+1}\right]$, the requests of $I$ are serviced according to $H_{i}$ (assume $s_{m+1}=\infty$ ). Each $H_{i}$ is an OffEndFit schedule for a suitable instance $I_{i}$. (So $H_{i}$ is just a "plan".) Let $m_{i}$ be the merit obtained by servicing $H_{i}$ in the time slot $\left(s_{i}, s_{i+1}\right]$. Then

$$
m e r i t(\operatorname{EndFit}(I))=\sum_{i=0}^{m} m_{i} .
$$

Now, there is a corresponding sequence of start times

$$
t_{0} \leq t_{1} \leq \cdots \leq t_{m-1} \leq t_{m}
$$

of requests in $\widetilde{I}$. Note that the $s_{i}$ 's and $t_{j}$ 's are basically identical except that $\operatorname{st}(q)$ in the list (6) is replaced by st $(\widetilde{q})$ in the list (8). We will make the lists (6) and (8) identical,

$$
s_{i}=t_{i} \quad(i=0, \ldots, m),
$$

simply by adding a null request with start time st $(\widetilde{q})$ into $I$, and by adding a null request with start time st $(q)$ into $\widetilde{I}$. Again, let $\widetilde{H}_{i}$ be the plan in the EndFit servicing of $\widetilde{I}$ at time $s_{i}$; so $\widetilde{H}_{i}$ is the OffEndFit schedule for a suitable instance $\widetilde{I}_{i}$. Let 
merit(EndFit $(\widetilde{I}))=\sum_{j=0}^{m} n_{j}$ be the equation for $\widetilde{I}$ corresponding to $(7)$. Our theorem follows if we show that $m_{i} \geq n_{i}$ for all $i$.

Let $i_{0}$ and $i_{1}$ be the indices such that $s_{i_{0}}=\operatorname{st}(q)$ and $s_{i_{1}}=\operatorname{st}(\widetilde{q})$. We may assume that $i_{0}<i_{1}$ : this is clear in case $\operatorname{st}(q)<\operatorname{st}(\widetilde{q})$. Even if $\operatorname{st}(q)=\operatorname{st}(\widetilde{q})$, we can arbitrarily assume $q$ started "just before" $\widetilde{q}$.

Now each $I_{i}$ is a residual instance of $I_{i-1}$, plus a new request $q_{i}$ that starts at time $s_{i}$. That is,

$$
I_{i}=\left.\left(I_{i-1}\right)\right|_{s_{i}} \cup\left\{q_{i}\right\}
$$

We say that $q_{i}$ is "inserted" into $I_{i}$. In case $i=i_{1}$, the request $q_{i}$ is a suitable null request. Similarly,

$$
\widetilde{I}_{i}=\left.\left(\widetilde{I}_{i-1}\right)\right|_{s_{i}} \cup\left\{\widetilde{q}_{i}\right\}
$$

where $\widetilde{q}_{i}$ is null request in case $i=i_{0}$.

Our goal of showing $m_{i} \geq n_{i}$ can be reduced to showing $I_{i}$ dominates $\widetilde{I}_{i}$. But for the induction to carry through, we need to maintain stronger assertion (via Lemma 10), that for all $i$,

$$
I_{i} \succeq \widetilde{I}_{i}
$$

It is not hard to see that (10) holds for all $i<i_{1}$. In fact, $I_{i}=\widetilde{I}_{i}$ for $i<i_{0}$. More generally, for all $i$ except $i=i_{1}$, the request $\widetilde{q}_{i}$ inserted into $\widetilde{I}_{i}$ is a trimmed version of the request $q_{i}$ inserted into $I_{i}$. By an application of Lemma 8 and Lemma 9, we conclude from $I_{i} \succeq \widetilde{I}_{i}$ that $I_{i+1} \succeq \widetilde{I}_{i+1}$.

It remains to show that $(10)$ holds when when $i=i_{1}$. Write $J_{1}$ for $\left.\left(I_{i_{1}-1}\right)\right|_{s_{1}}$ and $\widetilde{J}_{1}$ for $\left.\left(\widetilde{I}_{i_{1}-1}\right)\right|_{s_{1}}$. Since $I_{i_{1}-1} \succeq \widetilde{J}_{i_{1}-1}$, we conclude that

$$
J_{1} \succeq \widetilde{J}_{1}\left(\operatorname{via} f_{1}\right)
$$

where $f_{1}$ is naturally defined. In $J_{i_{1}}$ we have a request $r$ that could be traced back to $q=q_{i_{0}}$ at time $s_{i_{0}}$ (so $r$ is really $\left.q\right|_{s_{1}}$ ). Also, $f_{1}(r)$ is traced back to the null request that we inserted into $\widetilde{I}$ in order to ensure (9). We want to show that $I_{i_{1}} \succeq \widetilde{I}_{i_{1}}$ via the natural $f$ which extends $f_{1}$. Unfortunately, $f$ maps a null request, $q_{i_{1}}$, to $\widetilde{q}=\widetilde{q}_{i_{1}}$. But we had noted that $f_{1}$, and hence $f$, maps $r$ to a null request $f_{1}(r)$. We will modify $f$ so that $r$ is mapped to $\widetilde{q}$ and null request $q_{i_{1}}$ is mapped to $f_{1}(r)$. This change amounts to transposing $r$ and $q_{i_{1}}$ in $I_{i_{1}}$, and is harmless. We now claim that $I_{i_{1}} \succeq \widetilde{I}_{i_{1}}$ via $f$. In view of (11), we only need to show that $r$ and $f(r)=\widetilde{q}$ are related as required by the $\succeq$ relation. There are two cases: (a) If $q$ has not been served by EndFit $(I)$ up to time $s_{1}$ then $\widetilde{q}$ is just a trimmed version of $r$. (b) If EndFit $(I)$ had already started serving $q$ by time $s_{1}$, then $r$ is saturated in $I_{i_{1}}$. It is also obvious that $r$ spans $\widetilde{q}$. Q.E.D.

\subsection{EndFit is 2-competitive}

We now use Theorem 11 to show that EndFit is 2-competitive. 
Theorem 12 For any instance I,

$$
\operatorname{merit}(\operatorname{opt}(I)) \leq 2 \cdot \operatorname{merit}(\operatorname{EndFit}(I)) .
$$

Proof. We can assume that $I$ is intact in opt $(I)$. Let $\widetilde{I}$ be the trimmed instance of $I$ such that for any request $q \in I$, if $(\operatorname{opt}(I))^{-1}(q)=\left(t_{1}, t_{2}\right]$, then the corresponding trimmed request $\widetilde{q}$ satisfies st $(\widetilde{q}):=t_{1}$ and $\mathbf{s z}(\widetilde{q}):=t_{2}-t_{1}$; otherwise if opt $(I)^{-1}(q)=$ $\emptyset$, then $\widetilde{q}$ satisfies $\operatorname{sz}(\widetilde{q}):=0$. We can further assume that $\widetilde{I}$ is intact in all the plans of EndFit with $\widetilde{I}$. By definition, we have

$$
\operatorname{merit}(\operatorname{opt}(I))=\operatorname{merit}(\operatorname{opt}(\widetilde{I})) .
$$

The instance $\widetilde{I}$ has the nice property that requests arrive at a "constant rate", that is, if a request $q$ starts at time $t$, then no other request starts during $(t, t+\mathbf{s z}(q))$. Let $H_{1}$ and $H_{2}$ be two identical copies of EndFit $(\widetilde{I})$. Our theorem is proved if we show how to charge requests in opt $(\widetilde{I})$ to $H_{1}$ and $H_{2}$.

Consider a request $q$ in $\widetilde{I}$. Upon arrival of $q$, there are two cases.

1. If $q$ is allocated in the new plan $\operatorname{Plan}^{+}($EndFit, $\widetilde{I}, q)$, then it is possible that there are some requests which are originally allocated in $\operatorname{Plan}^{-}$(EndFit, $\widetilde{I}, q$ ), but not in the new plan. Call these requests the ousted requests.

2. Otherwise, call $q$ the ousted request.

Let $s$ be the total size of the ousted requests. Note that $s \leq \mathbf{s z}(q)$ and the total merit of the ousted requests is not more than the total merit of the requests allocated in $(\operatorname{st}(q), \operatorname{st}(q)+s]$ in $\operatorname{Plan}^{+}(\operatorname{EndFit}, \widetilde{I}, q)$. Furthermore, the new plan will be carried out without interruption at least until st $(q)+\mathbf{s z}(q)$. Charge the ousted requests to $H_{2}$ at $(\mathbf{s t}(q), \mathbf{s t}(q)+\mathbf{s z}(q)]$ and the served requests during $(\mathbf{s t}(q), \mathbf{s t}(q)+\mathbf{s z}(q)]$ to $H_{1}$ at $(\operatorname{st}(q), \mathbf{s t}(q)+\mathbf{s z}(q)]$. The above is a valid charging scheme. Thus, we have

$$
2 \cdot \operatorname{merit}(\operatorname{EndFit}(\widetilde{I})) \geq \operatorname{merit}(\operatorname{opt}(\widetilde{I})) \text {. }
$$

By Theorem 11 and (12), we have

$$
2 \cdot \operatorname{merit}(\operatorname{EndFit}(I)) \geq \operatorname{merit}(\operatorname{opt}(I)) .
$$

Q.E.D.

\section{A Class of Greedy Schedulers}

Looking at the behavior of EndFit and FirstFit on specific examples, it appears that they are complementary in the sense that if EndFit performs poorly on an 
instance, then FirstFit will perform well, and vice-versa. This suggests studying some combination of these two heuristics and motivates the generalization to a class of Greedy schedulers.

A scheduler $S$ in this class behaves as follows.

(A) At the moment a new request $q$ starts, it suspends the current service (that is, it preempts the currently served request).

(B) Scheduler $S$ computes a new plan $H$, which is a schedule for the set of residues of currently pending requests. We call $H$ a 'plan' because the scheduler may not carry out the schedule as planned due to the subsequent new requests. The plan $H$ is computed by considering the residues one by one, starting from the heaviest request down to the lightest request. Let $p$ be the request being considered and call $\left|H^{-1}(p)\right|$ the allocation to $p$. The allocation to $\mathrm{p}$ is subjected to the following restriction:

(*) The allocation to $p$ must be maximized. For example, if it is possible to completely allocate $p$, the whole of $p$ must be allocated. However, there is no restriction on where $p$ is allocated. Time-slots, once allocated, are not subsequently revised in creating this plan.

(C) It carries out the plan until a new request starts, whereupon we go back to step (A).

Let the plan computed after step (B) be $\operatorname{Plan}^{+}(S, I, q)$ and $\operatorname{Plan}^{-}(S, I, q)$ be the original plan just before step (B) is executed. Thus $\operatorname{Plan}^{-}(S, I, q)$ is actually $\operatorname{Plan}^{+}\left(S, I, q^{\prime}\right)$, where $q^{\prime}$ is the request which starts just before $q$.

Different members of the Greedy class differ only in their strategies for (B) subjected to the restriction $(*)$. Note that our first example FirstFit is a Greedy scheduler: the request $p$ in $(*)$ is allocated in the earliest possible time-slots.

The second example EndFit is also a greedy scheduler. Its strategy for (B) is rather counter-intuitive: the request $p$ is allocated in the latest possible time slots.

By combining the counter examples for FirstFit and EndFit, we can find a Greedy scheduler whose competitive ratio $\geq 3$. We state, without proof, the following result.

Theorem 13 Every Greedy scheduler is 3-competitive.

By further imposing a constraint on the greedy schedulers, it is possible to show that the restricted greedy schedulers have competitive ratio not better than 2 . Specifically, consider the grid-points at $N \epsilon$ where $N$ is an integer and $\epsilon>0$ is any small constant. The restricted greedy schedulers always allocate requests to time slots starting and ending at the grid-points. To show that a schedulers $S$ has competitive ratio no better than 2 , we construct a request $q_{1}=(0,2,1,1)$ and observe how $S$ 
schedulers $q_{1}$. Let $(s, t]$ be the earlies time slot where $S$ plans for $q_{1}$. If $s=0$, we introduce two requests $q_{2}=(s, t,(t-s), 1)$ and $q_{3}=(t, t, 0,0)$. Thus, within time slot $(s, t], S$ is forced to serve $q_{1}$ and it will be impossible to serve $q_{2}$ in the future. The request $q_{3}$ forces $S$ to preempt and compute a new plan at time $t$. If $s>0$, we introduce a request $q_{2}^{\prime}=(s, s, 0,0)$. Thus at time $s$, the scheduler $S$ is forced to preempt and recompute a plan. By repeating the process, we can obtain a counter example $I$ where merit $(\operatorname{opt}(I))=2$ whereas $\operatorname{merit}(S(I))=1$.

\section{General Lower Bound}

From the previous section, we know that every greedy scheduler has competitive ratio that lies between 2 and 3. Are there schedulers outside the Greedy class with competitive ratio less than 2? We note a partial result in this direction: every deterministic scheduler has competitive ratio at least $2(2-\sqrt{2})>1.17$.

In proof, consider this adversary: at time 0 , the adversary releases two requests $q_{0}:=(0,2,1,1)$ and $q_{1}:=(0,1,1, \sqrt{2}-1)$. At time $t=1$, let the residual size of $q_{0}$ be $s_{0}$. If $s_{0}$ is less than $1 / 2$ then the request $q_{2}:=(1,2,1,1)$ is released. Otherwise, no further requests will be released.

It may be verified that that any deterministic scheduler achieve a merit of at most $\frac{1}{2(2-\sqrt{2})}$ of the maximum possible.

Unfortunately, this lower bound of 1.17 leaves a wide gap from the current upper bound of 2 . On the other hand, no simple variation of this adversary seems to give a better lower bound.

\section{On the Number of Breakpoints and Multi-tasking}

Both FirstFit and EndFit make $O(n)$ breakpoints where $n$ is the number of requests. Does the number of breakpoints affect the performance of a scheduler? We give an alternative formulation of the scheduling problem which could be viewed as allowing an infinite number breakpoints. In this multi-tasking environment, several requests can be served concurrently, but each at a (possibly) different rate. However, in any time interval of size $\Delta t$, the total size of requests served within this interval must not exceed $\Delta t$. Alternatively, we allow "fractional service" where the total service at any moment sums to 1.

A concrete example of such a scheduler is FirstEndFit: It simulates FirstFit and EndFit concurrently and serves half of what FirstFit and EndFit would serve. That is, if FirstFit and EndFitwill serve $q$ and $p$ respectively in the time slot $\left(s_{0}, t_{0}\right]$, then FirstEndFit will serve $p$ and $q$ concurrently but each at half the rate. We suspect that FirstEndFit is (3/2)-competitive.

Note that FirstEndFit can be also viewed as the following randomized scheduler under the original single-tasking setting: 
1. Before receiving any request, it tosses a fair coin.

2. If the outcome is head, then it simulates FirstFit, otherwise it simulates EndFit.

Clearly, the expected merit gained by this randomized scheduler is same as the merit gained by FirstEndFit. In general, we can show that any randomized single-tasking scheduler is equivalent to a deterministic multi-tasking scheduler. We omit the details.

\section{Conclusion}

We have formulated a "level-of-service" scheduling problem that arises naturally in our thinwire visualization applications. This formulation is also useful in real-time systems where quality of jobs can be traded-off for time. We have derived several competitive algorithms in this setting. We continue to investigate the many interesting questions that are open. Besides sharpening the results in the paper, we pose the following directions for further work: (1) Find optimal schedulers which are not restricted to be on-line. (2) Study the problem with other measures of merit (instead of multiplicative weights in this paper). (3) Introduce a model of penalty for preemption. (4) Introduce randomization.

\section{Acknowledgments}

We thank Estie Arkin and Yi-Jen Chiang for discussions about the problem.

\section{References}

[1] S. Albers. Competitive online algorithms. BRICS Lecture Series LS-96-2, BRICS, Department of Computer Science, University of Aarhus, September 1996.

[2] S. Albers and Jeffery Westbrook. A survey of self-organizing data structures. Research Report MPI-I-96-1-026, Max-Planck-Institut für Informatik, Im Stadtwald, D-66123 Saarbrücken, Germany, October 1996.

[3] E.-C. Chang, C. Yap, and T.-J. Yen. Realtime visualization of large images over a thinwire. In IEEE Visualization '97 (Late Breaking Hot Topics), pages 45-48, 1997. See CD proceedings of conference. Paper from

ftp://cs.nyu.edu/pub/local/yap/visual/thinwire.ps.gz.

[4] E.-C. Chang. Foveation Techniques and Scheduling Issues in Thinwire Visualization. PhD thesis, Department of Computer Science, New York University, May 1998. 
[5] J. A. Hoogeveen and A. P. A. Vestjens. Optimal on-line algorithms for singlemachine scheduling. Integer Programming and Combinatorial Opt., pages 404414, 1996.

[6] R. J. Lipton and A. Tomkins. Online interval scheduling. In Proc. 5th Annual ACM-SIAM Symp. on Discrete Algorithms, pages 302-311, 1994.

[7] M. Manasse, L.A. McGeoch, and D. Sleator. Competitive algorithms for server problems. In Proc. 20th Annual ACM Symposium on Theory of Computing, pages 322-333, 1988.

[8] L. A. McGeoch and D. D. Sleator, editors. On-Line Algorithms. DIMACS series in Discrete Mathematics and Theoretical Computer Science, volume 7. American Mathematical Society, 1992.

[9] D. Sleator and R. Tarjan. Amortized efficiency of list update and paging rules. Communications of the ACM, 28(2):202-208, 1985.

[10] G. J. Woeginger. On-line scheduling of jobs with fixed start and end times. Theoretical Computer Science, 130:5-16, 1994.

[11] F. Yao, A. Demers, and S. Shenker. A scheduling model for reduced cpu energy. Symposium on Foundations of Computer Science, pages 374-382, 1995. 